\title{
Padrão de Comprometimento Articular em Pacientes com Lúpus Eritematoso Sistêmico e sua Associação com Presença de Fator Reumatóide e Hiperelasticidade
}

\author{
Pattern of Joint Involvement in Patients with Systemic Lupus \\ Erythematosus and its Association with Rheumatoid \\ Factor and Hypermobility
}

\author{
Claudine Juliana Caznoch( ${ }^{(1)}$, Letícia Esmanhotto $^{(1)}$, Marília Barreto Silva $^{(2)}$, Thelma L. Skare ${ }^{(2)}$
}

\section{RESUMO}

Introdução: as manifestações articulares do lúpus eritematoso sistêmico (LES) são extremamente variadas, embora esta variabilidade nem sempre seja devidamente apreciada pelo reumatologista. Objetivo: este estudo tem por objetivo estudar a freqüência de aparecimento das diferentes formas de comprometimento articular no LES. Objetivou-se, também, procurar associações entre os diferentes padrões articulares com fator reumatóide (FR) e presença de hiperelasticidade. Material e Métodos: foram estudados 147 pacientes com LES para padrão de envolvimento articular, presença de FR e grau de hiperelasticidade calculado pelo índice de Beighton. Resultados: na população estudada, 53,1\% dos pacientes tinham queixas articulares na apresentação da doença. No decorrer da mesma, esta foi encontrada em 80,3\%. Artralgia isolada estava presente em 31,3\%, artrite aditiva em 36,1\% e artrite migratória em 12,9\%. Em 4,8\% dos casos, existia artrite deformante sendo rhupus em 2,1 \% e artropatia de Jaccoud em 2,7\%. Encontrou-se associação positiva entre presença de FR e artrite aditiva $(p<0,001)$. Não se conseguiu demonstrar associação entre o aparecimento de hiperelasticidade e artropatia de Jaccoud. Conclusão: as queixas articulares são extremamente comuns no LES, sendo mais freqüente a presença de artrite (aditiva e migratória). Existe maior positividade do FR em pacientes com artrite aditiva.

Palavras-Chave: lúpus eritematoso sistêmico, artrite, artralgia, rhupus, Jaccoud.

\begin{abstract}
Introduction: the articular manifestations of systemic lupus erythematosus are diverse and its whole spectrum is not fully appreciated by rheumatologists. Objective: this study aimed to assess the frequency of different forms of lupus joint involvement. It had also an objective of this study to determine the relationship of different joint patterns and rheumatoid factor and the occurrence of hypermobility. Material and Methods: we studied 147 lupus patients for pattern of joint involvement, rheumatoid factor and presence of hypermobility by Beighton index. Results: in the studied population $53.1 \%$ had joint complaints at diagnosis and $80.3 \%$ during the disease. Isolated arthralgia was present in $31.3 \%$; arthritis with additive pattern was found in $36.1 \%$ and with migratory pattern in $12.9 \%$. In $4.8 \%$ there was deforming arthritis: $2.1 \%$ with rhupus and 2.7 with Jaccoud's syndrome. We found a positive association between rheumatoid factor and additive arthritis $(p<0.001)$. No association could be demonstrated between hypermobility and Jaccoud arthropathy. Conclusion: joint complaints are very common specially the presence of arthritis (additive and migratory). There is a higher positivity of rheumatoid factor in patients with the additive form.
\end{abstract}

Keywords: systemic lupus erythematosus, arthritis, arthralgia, rhupus and Jaccoud.

\footnotetext{
Serviço de Reumatologia do Hospital Universitário Evangélico de Curitiba (HUEC). Recebido em 17/10/05. Aprovado, após revisão, em 21/05/06.

1. Residente do Serviço de Reumatologia do HUEC.

2. Professora Assistente de Reumatologia da Faculdade Evangélica do Paraná.

Endereço para Correspondência: Thelma L. Skare, Rua João Alencar Guimarães, 796, CEP 80310-420, Curitiba, PR, Brasil, e-mail: tskare@onda.com.br
} 


\section{INTRODUÇÃO}

O espectro clínico das manifestações articulares do lúpus eritematoso sistêmico (LES) é bastante variado, mas, devido a outras manifestações potencialmente graves, dificilmente estas se tornam a queixa dominante. Nesta enfermidade podem ser encontrados quadros de dor articular, rigidez, sinovites fugazes e artrites deformantes não-erosivas (artropatia de Jaccoud) ou erosivas (rhupus) ${ }^{(1-6)}$. Nos casos em que o processo inflamatório é evidente, é possível que os pacientes passem inicialmente por um diagnóstico de artrite reumatóide (AR) até que a expressão completa do LES se torne evidente.

No LES, todas as articulações (grandes e pequenas) podem estar afetadas, incluindo-se até as temporomandibulares. Porém, nota-se uma preferência para o envolvimento das articulações metacarpofalangianas e interfalangianas proximais ${ }^{(7)}$.

No presente estudo, procurou-se analisar as manifestações articulares do LES na população local. Ele tem por objetivo estimar a freqüência dos diferentes padrões articulares nos pacientes com LES e verificar a sua possível associação com positividade de fator reumatóide (FR) e ocorrência de hiperelasticidade articular.

\section{CASUÍSTICA E MÉTODOS}

Esta pesquisa foi aprovada pelo Comitê de Ética em pesquisa. Foram estudados os pacientes com LES que freqüentaram o ambulatório de Reumatologia do Hospital Universitário Evangélico de Curitiba (HUEC) durante o segundo semestre de 2004 e primeiro semestre de 2005, com pelo menos quatro critérios do American College of Rheumatology (ACR) para classificação desta doença e que concordaram em participar do estudo. Foram excluídos portadores de fibromialgia secundária, pacientes com hipotireoidismo não-controlado, portadores do vírus da hepatite $\mathrm{C}$ e os com insuficiência renal.

Os pacientes foram analisados quanto à presença de sintomas articulares ao início do quadro e no decorrer do mesmo. Tais sintomas foram classificados em artralgias sem artrite, artrites não-deformantes (sendo esta última subdividida em migratória ou aditiva) e artrites deformantes (tipo Jaccoud ou rhupus). Os diagnósticos de artrite e artralgia foram obtidos através da revisão dos prontuários, sendo admitidos como tais quando avaliados de maneira positiva ao exame físico pelo médico atendente. Pacientes com poliartrite aditiva e/ou presença de deformidades foram submetidos a estudos radiológicos das mãos. Foram considerados com Jaccoud aqueles com qualquer grau de desvios articulares redutíveis e sem erosões ao raio $\mathrm{X}^{(5)}$; com rhupus aqueles com deformidades irredutíveis e erosões detectadas ao raio $\mathrm{X}^{(3)}$ (Figura $1 \mathrm{~A}$ e B).
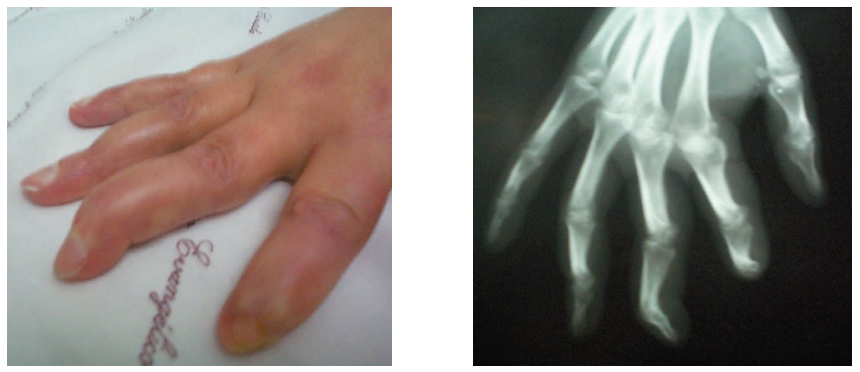

Figura $1 \mathrm{~A}$ - Foto de mãos e raio $\mathrm{X}$ de paciente com artropatia de Jaccoud
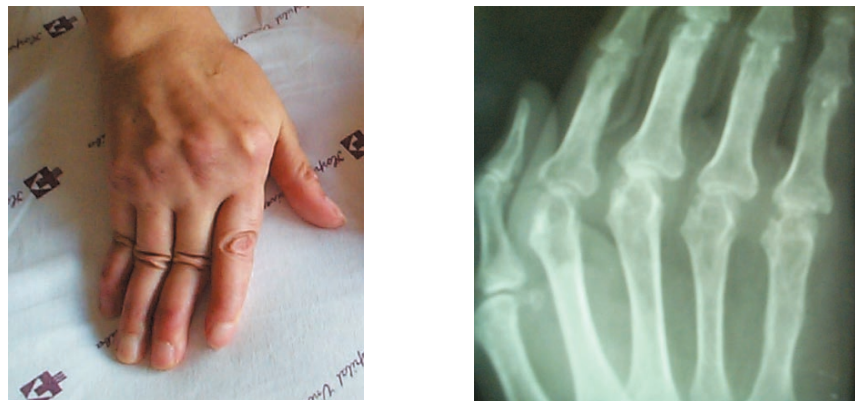

Figura 1 B - Foto de mãos e raio $\mathrm{X}$ de paciente com artropatia erosiva (rhupus)

Os pacientes foram submetidos à pesquisa do FR por imunoturbidimetria, sendo adotado como limite superior normal o de $15 \mathrm{UI} / \mathrm{ml}$. Em 97 destes pacientes, obteve-se o índice de Beighton, que é um índice destinado a analisar o grau de elasticidade $\operatorname{articular}^{(8)}$. O índice de Beighton é obtido atribuindo-se um ponto para: (a) capacidade de dorsiflexão do $5^{\circ}$ metacarpofalangiano a $90^{\circ}$, bilateralmente; (b) capacidade de aposição do polegar à face flexora do antebraço, bilateralmente; (c) hiperextensão do cotovelo acima de $90^{\circ}$, bilateralmente; (d) hiperextensão do joelho acima de $90^{\circ}$, bilateralmente; (e) capacidade de flexionar o tronco para frente e colocar a palma das mãos no chão. De acordo com esta escala, são considerados hiperelásticas as pessoas com valor acima de quatro pontos ${ }^{(8)}$.

Durante a medida do índice de Beighton, obteve-se a porcentagem das diferentes manifestações articulares presentes, sendo considerados portadores de artrite aqueles que apresentavam edema ao exame físico e de artralgia aqueles com sintomas dolorosos à palpação e mobilização da articulação. Foram anotados, também, os medicamentos em uso naquela ocasião. 
Os dados obtidos foram estudados por tabelas de freqüência e contingência, utilizando-se os testes de quiquadrado e Fisher, com auxílio do software GraphPad versão 2.0. Nível de significância adotado de 5\%.

\section{RESULTADOS}

Estudaram-se 147 pacientes. Sete eram homens e 140 eram mulheres. O diagnóstico de LES foi feito entre as idades de 5 e 68 anos (mediana de 30 anos) e estes pacientes estavam em acompanhamento no ambulatório do HUEC pelo período de 2 e 384 meses (mediana de 48 meses).

$\mathrm{O}$ estudo das manifestações articulares mostrou que em 78 pacientes (ou $53,1 \%$ ) existia a queixa articular como manifestação inicial da doença, e em 118 pacientes (ou $80,3 \%$ ) existia a queixa articular em algum tempo de sua enfermidade.

No decorrer da doença, a queixa mais comum foi a de artrite que apareceu em 48,9\% (72 pacientes), seguida pela de artralgia em $31,3 \%$ (46 pacientes). Dos pacientes com artrite, em 19 deles (ou 12,9\%) a forma era migratória e em 53 (ou 36,1\%) era aditiva. Existiam sete (ou 4,8\%) pacientes com artrite deformante, sendo três pacientes com rhupus $(2,1 \%)$ e quatro com artropatia de Jaccoud $(2,8 \%)$.

Veja as diferentes formas de artrite na população estudada no Gráfico 1.

\section{GrÁFICO 1}

\section{Presença de manifestações articulares durante a doença (147 pacientes)}

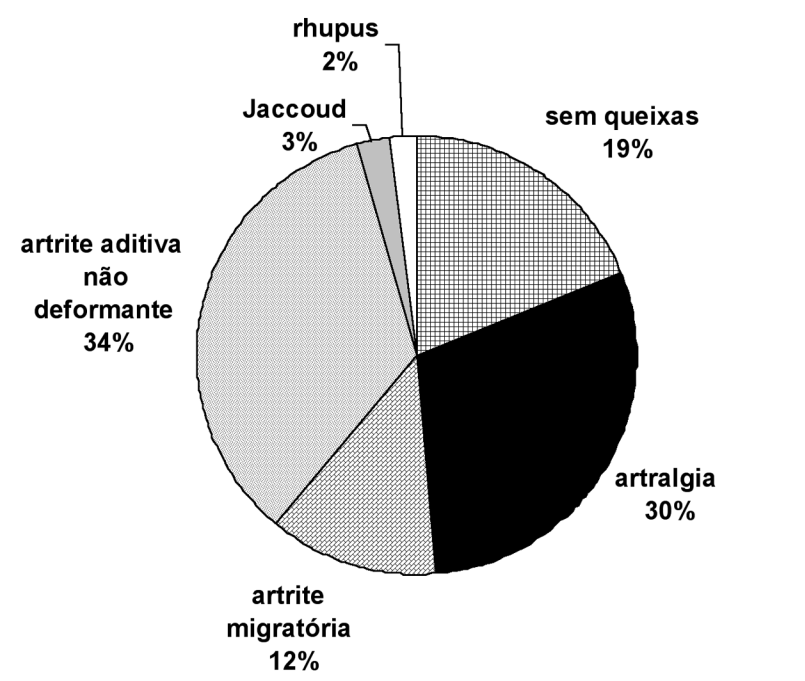

Obteve-se o teste do látex para FR em 112 pacientes, sendo este teste positivo em 30 deles (ou 26,8\%). Ao se estudar a associação entre FR positivo e sintomas articulares, obtiveram-se os dados resumidos na Tabela 1.

TABELA 1

Positividade Do FATOR REUMATÓIDE (FR), SEGUNDO O padrão de ENVOLVIMENTO ARTiCUlar ( $\mathrm{N}=112$ PACIENTES)

\begin{tabular}{|c|c|c|c|}
\hline & $\begin{array}{l}\text { FR positivo } \\
\quad(n=30)\end{array}$ & $\begin{array}{l}\text { FR negativo } \\
(n=82)\end{array}$ & $\mathrm{p}$ \\
\hline $\begin{array}{l}\text { Com sintomas } \\
\text { articulares }(n=94)\end{array}$ & $n=26(27,6 \%)$ & $\mathrm{n}=68(72,3 \%)$ & 0,775 (NS) \\
\hline Artralgia $(n=37)$ & $\mathrm{n}=9 \quad(24,3 \%)$ & $\mathrm{n}=28(75,6 \%)$ & 0,679 (NS) \\
\hline Artrite $(n=58)$ & $\mathrm{n}=17(29,3 \%)$ & $\mathrm{n}=42(72,4 \%)$ & 0,568 (NS) \\
\hline $\begin{array}{l}\text { Artrite migratória } \\
(\mathrm{n}=15)\end{array}$ & $\mathrm{n}=2(13,3 \%)$ & $n=13(86,6 \%)$ & $0,346(\mathrm{NS})$ \\
\hline Artrite aditiva $(n=43)$ & $\mathrm{n}=15(34,9 \%)$ & $\mathrm{n}=28(65,1 \%)$ & $<0,001(S)$ \\
\hline
\end{tabular}

$\mathrm{n}=$ número de pacientes da amostra; $\mathrm{NS}=$ não significante; $\mathrm{S}=$ significante.

$\mathrm{O}$ índice de Beighton foi medido em 97 pacientes, sendo que em 16 deles $(16,3 \%)$ existiam níveis acima de quatro, compatíveis com hiperelasticidade.

Estudando-se a associação entre índice de Beighton e diferentes formas de artrite, obtiveram-se os dados da Tabela 2.

\section{TABELA 2}

EsTUdo dE ASSOCIAÇ̃̃o ENTRE ÍNDICE DE BEIGHTON E TIPO DE ARTRITE NA POPULAÇÃO LÚPICA ( $\mathrm{N}=97)$

\begin{tabular}{|c|c|c|c|}
\hline & $\begin{array}{c}\text { Beighton }>4 \\
(n=16)\end{array}$ & $\begin{array}{c}\text { Beighton }<4 \\
(n=81)\end{array}$ & $\mathrm{p}$ \\
\hline $\begin{array}{l}\text { Com qualquer forma } \\
\text { de queixa articular }\end{array}$ & $n=13(56,25 \%)$ & $n=65(80,24)$ & $\mathrm{p}=1.0$ (NS) \\
\hline Com artralgias & $\mathrm{n}=2(12,5 \%)$ & $n=23(28,3 \%)$ & $\mathrm{p}=0.227(\mathrm{NS})$ \\
\hline $\begin{array}{l}\text { Com artrite } \\
\text { não-deformante } \\
\text { (migratória e aditiva) }\end{array}$ & $n=7 \quad(43,7 \%)$ & $\mathrm{n}=39(48,1 \%)$ & $\mathrm{p}=0.615$ (NS) \\
\hline Com artrite migratória & $\mathrm{n}=3(18,7 \%)$ & $\mathrm{n}=8(9,87 \%)$ & $\mathrm{p}=0.390(\mathrm{NS})$ \\
\hline Com artrite aditiva & $n=4(25 \%)$ & $n=31(38,2 \%)$ & $\mathrm{p}=0.102(\mathrm{NS})$ \\
\hline $\begin{array}{l}\text { Presença de } \\
\text { artrite deformante } \\
\text { (Jaccoud e rhupus) }\end{array}$ & $n=4(25 \%)$ & $\mathrm{n}=3(3,3 \%)$ & $\mathrm{p}=0.0127(\mathrm{~S})$ \\
\hline
\end{tabular}

n= número de pacientes da amostra; NS= não significante.

Dos 97 pacientes que se submeteram à determinação do índice de Beighton, $8(8,24 \%)$ tinham artralgia e outros $3(3,09 \%)$ tinham sinais flogísticos ao exame físico. Destes pacientes, todos (exceto 4 deles) faziam uso de difosfato de cloroquina (na dose de $4-7 \mathrm{mg} / \mathrm{kg} /$ dia de cloroquina), 16 
faziam uso de azatioprina (nas doses de 100 a $150 \mathrm{mg} / \mathrm{dia}$ ), 9 usavam metotrexato (nas doses de 7,5 a $20 \mathrm{mg} / \mathrm{semana}$ ), 1 fazia uso de ciclosporina (na dose de $150 \mathrm{mg} /$ dia), 1 de micofenolato mofetil ( $2 \mathrm{~g} /$ dia $)$ e 10 estavam em uso de pulsos de ciclofosfamida para manifestações renais e de sistema nervoso central (SNC) concomitantes. Prednisona estava sendo usada em doses entre de 5 e $80 \mathrm{mg} /$ dia em 68 deles. Das três pacientes com sinais flogísticos, duas estavam em uso de metotrexato e uma em uso de azatioprina, além de corticóide em doses de até $10 \mathrm{mg} /$ dia.

\section{DISCUSSÃO}

As queixas articulares no LES estão entre as que aparecem mais comumente, seja no início da doença, seja durante o decorrer da mesma. Segundo Dubois et a ${ }^{(7)}$, elas surgem em 33\% das formas iniciais de LES e em $90 \%$ dos casos durante o decorrer da sua história natural.

A classificação das queixas articulares no LES pode ser feita em: artralgias, artrites não-deformantes e artrites deformantes tipo Jaccoud (sem erosão ao raio X) ou tipo rhupus (com erosão ao raio $\mathrm{X})^{(5)}$. Veja Tabela 3 .

TABELA 3

ESPECTRO DAS MANIFESTAÇÕES ARTICULARES DO LES

Artralgias: \begin{tabular}{l} 
Artrites: $\left\{\begin{array}{l}\text { Não-deformantes; } \\
\text { Deformantes }\end{array} \quad\left\{\begin{array}{l}\text { Sem erosão ao raio X-Jaccoud; } \\
\text { Com erosão ao raio X-rhupus. }\end{array}\right.\right.$ \\
\hline
\end{tabular}

A denominação de artrite de Jaccoud para os casos de artrite deformante de mãos e pés, sem erosões ao raio $\mathrm{X}$, em pacientes lúpicos foi proposta por Bywaters, devido à semelhança com casos de artropatia de Jaccoud da moléstia reumática ${ }^{(5)}$. Nesta situação, as articulações sofrem desvios devido à frouxidão de ligamentos e cápsulas, além de fibrose periarticular. A deformidade tende a ser redutível e o raio X não apresenta doença erosiva, podendo demonstrar formações em "ganchos" nos metacarpianos ${ }^{(5,6)}$.

Babini et $a^{(9)}$ encontraram aumento de frouxidão ligamentar dos joelhos e casos de síndrome de Jaccoud em LES com insuficiência renal crônica, nos quais existia um hiperparatireoidismo secundário ${ }^{(9)}$. Estes autores atribuíram o fato de os ligamentos se tornarem enfraquecidos ao aumento do paratormônio - o que poderia explicar o aparecimento da artropatia de Jaccoud. Por outro lado, Gumà et $\mathrm{a}^{(10)}$ descreveram um aumento de frouxidão ligamentar e capsular em doentes com LES quando comparados com a população normal, sem associar o seu aparecimento a qualquer característica clínica, exceto maior tempo de doença e uso de corticóide.

Já a denominação de rhupus pressupõe um quadro misto de LES e $\mathrm{AR}^{(11)}$. Aliás, muito tem sido debatido sobre a possibilidade deste quadro representar uma associação das duas enfermidades (AR e LES) ou, apenas, um subgrupo dos pacientes com LES. A possibilidade de co-existência das duas enfermidades tem sido estimada em $0,09 \%^{(3)}$, um valor mais baixo do que o habitualmente encontrado. Van Vught et $a^{\left({ }^{5}\right)}$ encontraram uma prevalência de artrite erosiva em 1\% de 176 pacientes. Por outro lado, Reilly et al ${ }^{(1)}$ encontraram erosões em até $27,5 \%$ de 51 pacientes lúpicos submetidos a estudos radiológicos. A maioria dos autores admite, no entanto, que o rhupus é uma expressão clínica do LES ao invés da sua associação com $\operatorname{AR}^{(11)}$.

Segundo Alarcón-Segovia et a ${ }^{4}{ }^{4}$, os pacientes de LES com artropatia deformante (com e sem erosões) têm maior prevalência de FR. Estes autores levantaram a possibilidade da participação deste auto-anticorpo nas deformidades através de formação de complexos imunes. Ainda segundo estes mesmos pesquisadores, tais pacientes teriam também uma maior prevalência de síndrome seca e uma menor proporção de fotossensibilidade e eritema malar.

Encontrou-se, no presente estudo, uma alta positividade de manifestações articulares, o que está de acordo com dados previamente estabelecidos. Entretanto, neste grupo, a ocorrência de artrite (aditiva e migratória) foi maior do que a de artralgias isoladas. Interessantemente, a ocorrência de artrite aditiva foi maior nos pacientes com FR positivo - o que nos ajuda a compreender as dificuldades de diagnóstico diferencial entre LES e AR no início da doença. Se a positividade do FR influi de alguma maneira nesta forma de apresentação, é assunto a ser melhor analisado.

Não se conseguiu demonstrar associação entre hiperelasticidade e qualquer forma de comprometimento articular, exceto com as artrites deformantes em geral. Devido ao baixo número de pacientes com Jaccoud e com rhupus na presente amostra, não foi possível apreciar de maneira adequada o seu aparecimento nas diferentes formas de artrite deformante. Estudos com um maior número de pacientes seriam interessantes para verificar uma possível implicação da hiperelasticidade no aparecimento de artrite de Jaccoud.

A influência do sexo dos pacientes nas variáveis estudadas também não pode ser avaliada uma vez que a amostra se compunha de apenas sete pacientes do sexo masculino. 


\section{CONCLUSÃO}

Em pacientes com LES, encontrou-se uma alta freqüência de manifestações articulares: $53,1 \%$ no início da doença e $80,3 \%$ no decorrer da mesma.

A forma de comprometimento articular mais comum foi de artrite aditiva.

Encontrou-se associação entre a presença de FR e a ocorrência de artrite aditiva.

A ocorrência de hiperelasticidade só foi significativa nos

\section{REFERÊNCIAS}

1. Reilly PA, Evison G, McHugh NJ, Maddison PJ: Arthropathy of hands and feet in systemic lupus erythematosus. J Rheumatol 17: 777-84, 1990.

2. Sczepanski L, Targonskaiotrowski M: Deforming arthropathy and Jaccoud's syndrome in patients with systemic lupus erythematosus. Scand J Rheumatol 21: 308-9, 1992.

3. Pannush RS, Edwards L, Longley S, Webster E: Rhupus' syndrome. Arch Intern Med 148: 1633-6, 1988.

4. Alarcón-Segovia D, Abud-Mendoza C, Diaz-Jouanen E, Iglesias A, De los Reyes V, Hernandez-Ortiz J: Deforming arthropathy of the hands in systemic lupus erythematosus. J Rheumatol 15: 65-9, 1988.

5. Van Vugt RM, Derksen RHWM, Kater L, Bijlsma JWJ: Deforming arthropathy or lupus hands in systemic lupus erythematosus. Ann Rheum Dis 57: 540-4, 1998.

6. Spronk PE, Borg EJ, Kallenberg CGM: Patients with systemic lupus erythematosus and Jaccoud's arthropathy: a clinical subset portadores de formas deformantes. A análise da possível associação de hiperelasticidade com a forma de Jaccoud não pode ser devidamente avaliada, dada a baixa freqüência de aparecimento desta forma de comprometimento articular. São necessários maiores estudos para verificar se a presença de hiperelasticidade influi, ou não, no aparecimento da artropatia de Jaccoud.

Declaramos a inexistência de conflitos de interesse. with an increased C reative protein response? Ann Rheum Dis 51: 358-61, 1992.

7. Dubois EL, Wallace DJ: Clinical and laboratory manifestations of systemic lupus erythematosus. In: Wallace DJ, Dubois EL (Eds). Dubois's Lupus Erythematosus, $3^{\text {rd }}$ ed, Philadelphia, Lea \& Febiger, 312-449, 1987.

8. Grahame R: Hypermobility syndrome. In Klippel JH, Dieppe PA (Eds). Rheumatology, $2^{\text {nd }}$ ed, London, Mosby, S-8: 51.1- 6 , 1998.

9. Babini SM, Cocco MJA, de la Sota M, Arturi JCM, Marcos JC, Morteo OG: Tendinous laxity and Jaccoud's syndrome in patients with systemic lupus erythematosus possible role of secondary hyperparathyroidism. J Rheumatol 16: 494-8, 1989.

10. Gumà $\mathrm{M}$, Olive $\mathrm{A}$, Roca $\mathrm{J}$ et al: Association of systemic lupus erythematosus and hypermobility. Ann Rheum Dis 61: 1024-6, 2002.

11. Fernández A, Quintana G, Matteson EL, Restrepo JF, Rondón F, Sanchez A, Iglesias A: Lupus arthropathy: hystorical evolution from deforming arthritis to rhupus. Clin Rheumatol 23: 523-6, 2004. 